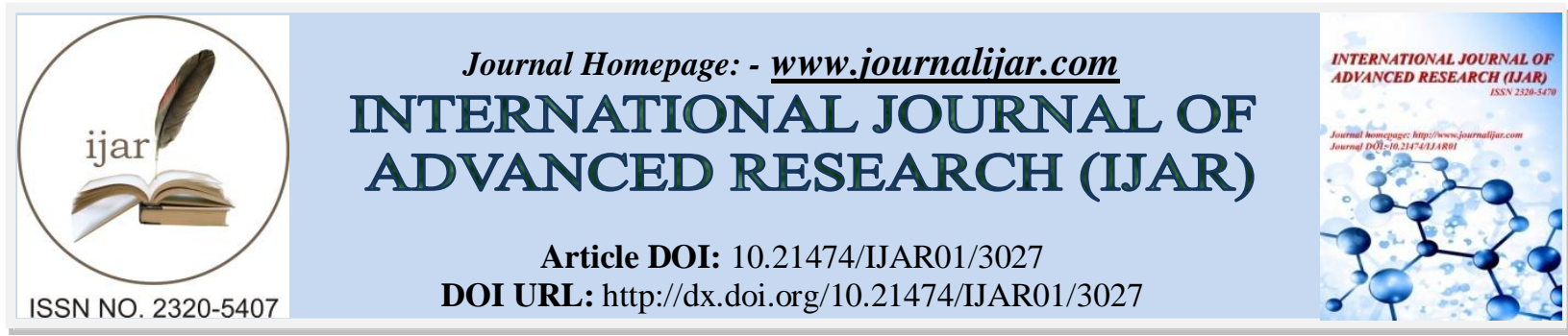

RESEARCH ARTICLE

\title{
THE USE OF THE MEMBRANE - REDOX POTENTIALS THREE - STATE LINE SYSTEM DEPENDENT - FULL 9 STEPPED CYCLE OF PROTON CONDUCTANCE - RELATED REGULATIONS IN THE ANTICANCER TREATMENT.
}

\author{
M. Ambaga. \\ Mongolia, Ulanbator, New medicine medical institute.
}

\section{Manuscript Info}

\section{Manuscript History}

Received: 29 November 2016

Final Accepted: 27 December 2016

Published: January 2017

\section{Key words:-}

The membrane - redox potentials three state line system dependent - full 9 stepped cycle of proton conductance, the solid betta state, the fluid alpha state, the gamma state

\section{Abstract}

The essence of our new idea in relating to how to decrease the intensity of cancer cell growth by changing and establishing the right balance between glycolysis process and the process of proton, electron conductance in the membrane - redox potentials three - state line system dependent - full 9 stepped cycle, which functioned with using of oxygen is follows:

a. Maintaining of normal intensity of glycolysis process by stimulation the proton, electron conductance in the 2-th stage of the full 9 stepped cycle of proton conductance inside human body in the level of isocitrate dehydrogenase, alpha ketoglutarate dehydrogenase and succinate dehydrogenase.

b. Maintaining of normal intensity of glycolysis process by stimulation of the formation of oxaloacetate from malate under action of malate dehydrogenase in the 2-th stage of the full 9 stepped cycle of proton conductance inside human body in the level of isocitrate dehydrogenase, alpha ketoglutarate dehydrogenase and succinate dehydrogenase.

1-th stage of the full 9 stepped cycle of proton conductance inside human body is distinguished by release of proton, electron from food substrates (carbohydrate, amino acids, fatty acids), under the undirect action of oxygen released from membrane surroundings of erythrocyte in the 9 stage, from this stage started the proton conductance within cycle.

The maintaining of increased fluid alpha states, consisting of unsaturated fatty acids with high levels of oxy potentials conducting the flow of protons and electrons in this stage of cancer cells more useful to decrease the cancer cell growth.

If needed to decrease the cell division rates during cancer should be increased the alpha state with high oxidizing potentials in the reaction medium of "Donators + membrane - redox potentials three - state line system $+\mathrm{O}_{2}+\mathrm{ADP}+\mathrm{Pi}+\mathrm{H}^{+}+\mathrm{nH}+$ membrane space $=(\mathrm{ATP}+$ heat energy) $+\mathrm{H}_{2} \mathrm{O}+\mathrm{nH}+$ matrix $+\mathrm{CO}_{2}$ "

Copy Right, IJAR, 2016,. All rights reserved. 


\section{Introduction:-}

Untill now we can not drive and regulate the intensity of metabolism reaction during cancer to more usefull needed direction because we do not know in which place of cells are existed driving point of regulations subjected to action of such kinds of manipulation and medicaments.

Revealed by us the membrane - redox potentials three - state line system is one of more important member of proposed by us the full 9 stepped cycle of proton conductance inside the human body and one of these places of cells, which easily subjected to action of manipulation and medicaments as driving point to conduct the usefull favorable direction of metabolism reactions during cancer, diabetes mellitus and hypercholesterinemia.

In such a case, membrane - redox potential's three - state line system serves the role of main driver.

We can create all the variants of useful reaction background forms of metabolism by changing the membrane redox potentials three - state line system of the full 9 stepped cycle of proton conductance inside the human body.

If needed to decrease the cell division rates during cancer should be increased the alpha state with high oxidizing potentials in the reaction medium of "Donators + membrane - redox potentials three - state line system $+\mathrm{O}_{2}+$ ADP $+\mathrm{Pi}+\mathrm{H}^{+}+\mathrm{nH}+$ membrane space $=(\mathrm{ATP}+$ heat energy $)+\mathrm{H}_{2} \mathrm{O}+\mathrm{nH}+$ matrix $+\mathrm{CO}_{2} "$

\section{Results and conclusion:-}

We proposed that the biochemical characteristics of cancer cells more similar to Archea (prokaryotic) as if cancer cells acquired the some feature of Archea, as if normal cells made the evolutionary back steps to direction of the early evolution cells (as if Eukarya cells turn to Archaea- prokaryotic cells) with whom could be meet 4,4 billion years ago according to Woese classification .

The Archaea adapted to exist in extreme environments, niches devoid of oxygen and whose temperatures can be near or above the normal boiling point of water.

Archaea are prokaryotic cells which are typically characterized by solid membrane lipids that are branched hydrocarbon chains attached to glycerol by ether linkages. The presence of these ether linkages in Archaea adds to their ability to withstand extreme temperatures and highly acidic conditions.

We can see abovementioned feature of Archea, possessing the solid betta state of membrane structures and their ability to withstand extreme temperatures and highly acidic conditions in example of cancer cells, behind these regulations may be existed the such lawfull process as rapidly growing tumor cells typically have glycolytic rates up to 200 times higher than those of their normal tissues of origin, this occurs even if ox ygen is plentiful.

The Warburg effect is the observation that most cancer cells predominantly produce energy by a high rate of glycolysis .

Otto Warburg postulated this change in metabolism as glycolysis, is the fundamental cause of cancer.

Many substances have been developed which inhibit glycolysis, and such inhibitors are currently the subject of intense research as anticancer agents.

In this connection raised the questions that if most cancer cells produce energy by a high rate of glycolysis in the cytosol, what kinds of positive usefull changes should be induced in the membrane - redox potentials three state line system dependent - full 9 stepped cycle of proton conductance because glycolysis followed by oxidation of donators in this cycle. 


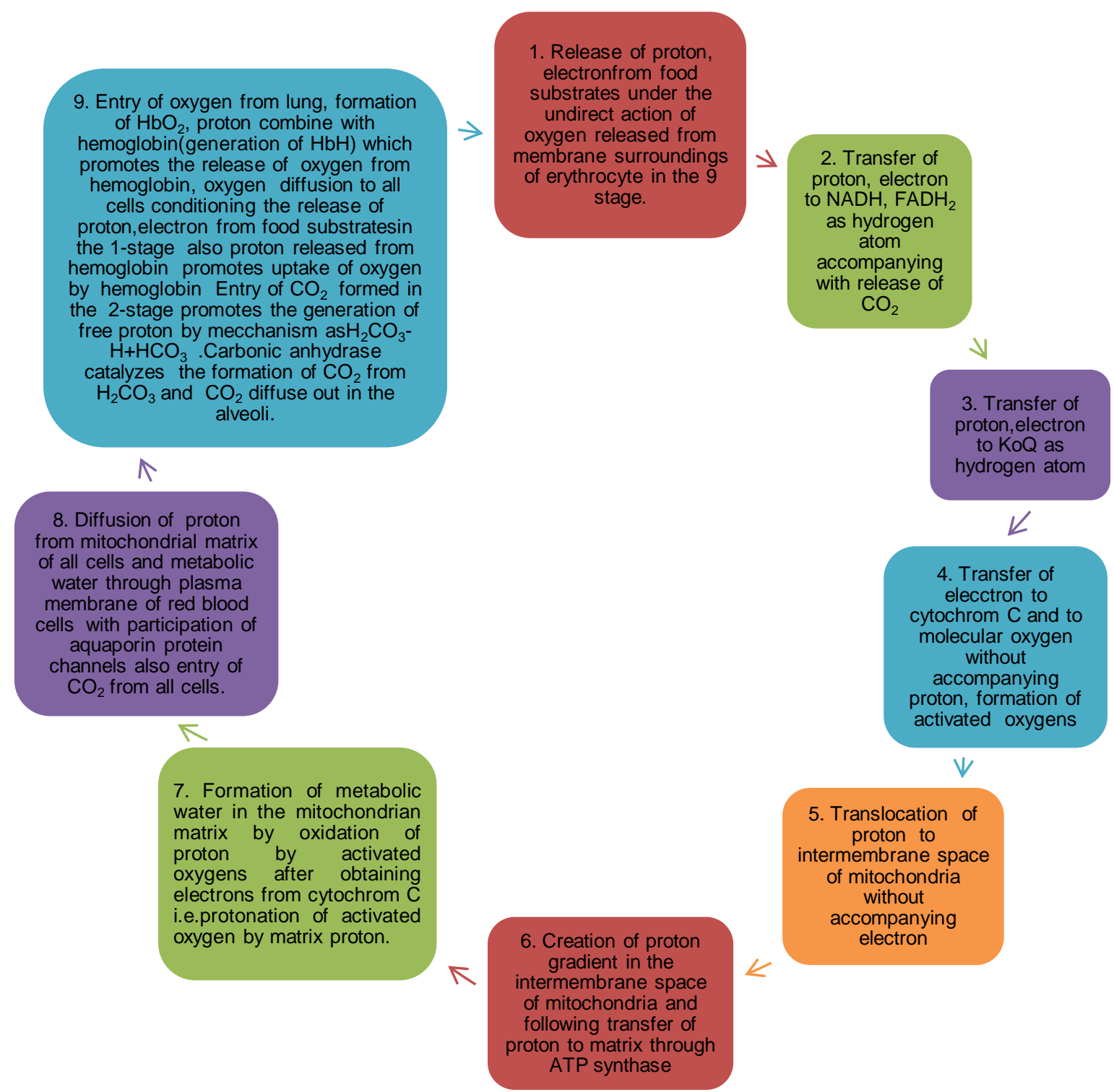

Also raised the questions how to use this positive usefull changes in the anticancer treatment by causing the usefull shift in the the membrane - redox potentials three - state line system dependent - full 9 stepped cycle of proton conductance such as from the solid betta state of membranes consisting of mainly saturated fatty acids conditioning a high levels of red potentials to the fluid alpha state of membranes, consisting of unsaturated fatty acids with high levels of oxy potentials, also from the gamma state of membranes consisting of decreased contents of saturated unsaturated fatty acids, conditioning a decreased levels of redoxy potentials to the fluid alpha state of membranes with high levels of oxy potentials.

9-th stage of the the membrane - redox potentials three - state line system dependent - full 9 stepped cycle of proton conductance is distinguished by entry of oxygen from lung, formation of $\mathrm{HbO}_{2}$, proton combine with hemoglobin (generation of $\mathrm{HbH}$ ) which promotes the release of oxygen from hemoglobin, oxygen diffusion to all cells conditioning the release of proton, electron from food substrates.

The abovementioned explanation shows that promotion of the release of oxygen from hemoglobin, oxygen diffusion to all cells conditioning the release of proton, electron from food substrates in the 9-th stage of the the membrane redox potentials three - state line system dependent - full 9 stepped cycle of proton conductance by increasing the fluid alpha state of membranes, consisting of unsaturated fatty acids with high levels of oxy potentials in cancer cells may be used in the anticancer treatment. 


\section{We proposed that:-}

The essence of our new idea in relating to how to decrease the intensity of cancer cell growth by changing and establishing the right balance between glycolysis process and the process of proton, electron conductance in the membrane - redox potentials three - state line system dependent - full 9 stepped cycle, which functioned with using of oxygen is follows:

a. Maintaining of normal intensity of glycolysis process by stimulation the proton, electron conductance in the 2-th stage of the full 9 stepped cycle of proton conductance inside human body in the level of isocitrate dehydrogenase, alpha ketoglutarate dehydrogenase and succinate dehydrogenase.

b. Maintaining of normal intensity of glycolysis process by stimulation of the formation of oxaloacetate from malate under action of malate dehydrogenase in the 2-th stage of the full 9 stepped cycle of proton conductance inside human body in the level of isocitrate dehydrogenase, alpha ketoglutarate dehydrogenase and succinate dehydrogenase.

1-th stage of the full 9 stepped cycle of proton conductance inside human body is distinguished by release of proton, electron from food substrates (carbohydrate, amino acids, fatty acids), under the undirect action of oxygen released from membrane surroundings of erythrocyte in the 9 stage, from this stage started the proton conductance within cycle.

The maintaining of increased fluid alpha states, consisting of unsaturated fatty acids with high levels of oxy potentials conducting the flow of protons and electrons in this stage of cancer cells more useful to decrease the cancer cell growth.

2-th stage of the full 9 stepped cycle of proton conductance inside human body is distinguished by transfer of proton, electron to $\mathrm{NADH}, \mathrm{FADH}_{2}$ as hydrogen atom accompanying with release of $\mathrm{CO}_{2}$, by which stage continued the proton conductance within cycle.

The maintaining of increased fluid alpha states, consisting of unsaturated fatty acids with high levels of oxy potentials conducting the flow of protons and electrons in this 2-th stage of cancer cells more useful to decrease the cancer cell growth.

4-th stage of the full 9 stepped cycle of proton conductance inside human body is distinguished by transfer of electron to cytochrom $\mathrm{C}$ without accompanying proton, which one is stages of continuity of the proton conductance within cycle and the increase of the fluid alpha state, consisting of unsaturated fatty acids with high levels of oxy potentials conducting the flow of protons and electrons.

The maintaining of increased fluid alpha states, consisting of unsaturated fatty acids with high levels of oxy potentials conducting the flow of protons and electrons in this 4-th stage of cancer cells more useful to decrease the cancer cell growth.

According to the full 9 stepped cycle of proton conductance inside human body proposed by Ambaga and TumenUlzii (2015) 6- th stage of the full 9 stepped cycle of proton conductance inside human body is distinguished by creation of proton gradient in the intermembrane space of mitochondria and following transfer of proton to matrix through ATP synthase, which is played important role in continuity of the proton conductance within cycle.

The maintaining of increased fluid alpha states, consisting of unsaturated fatty acids with high levels of oxy potentials conducting the flow of protons and electrons in this 6- th stage of cancer cells more useful to decrease the cancer cell growth.

Also 7- th stage of the full 9 stepped cycle of proton conductance inside human body is distinguished by formation of metabolic water in the mitochondrian matrix by oxidation of proton by molecular oxygens i.e, by protonation of molecular oxygen by matrix proton, which is one of a previous stages of continuity of the proton conductance cycle and in this connection the increase of the fluid alpha state, consisting of unsaturated fatty acids with high levels of oxy potentials conducting the flow of protons and electrons in this 7 - th stage of cancer cells more useful drcrease the cancer cell growth. 
9-th stage is distinguished by entry of oxygen from lung, formation of $\mathrm{HbO}_{2}$, proton combine with hemoglobin (generation of $\mathrm{HbH}$ ) which promotes the release of oxygen from hemoglobin, oxygen diffusion to all cells conditioning the release of proton, electron from food substrates and the increase of the fluid alpha state, consisting of unsaturated fatty acids with high levels of oxy potentials conducting the flow of protons and electrons.

The maintaining of increased fluid alpha states, consisting of unsaturated fatty acids with high levels of oxy potentials conducting the flow of protons and electrons in this 9-th stage of cancer cells more useful to decrease the cancer cell growth.

\section{References:-}

1. Ambaga M, Tumen-Ulzii A (2016). Integrated NCM medicine with s-NCM new knowledge, lambert Academic Publishing

2. Ambaga M, Tumen-Ulzii A (2015). The life become dependent from the presence of electrons and protons, which were formed during events called big bang 15 billion years ago, electrons and protons sets the stage for formation of life in the universe

3. Ambaga M (2016). The Full Cycle of Proton and Electron Conductance inside the Human Body, Consisting of 9 Linked Stages. Acad. J. Sci. Res. 4(6): 127-131.

4. Ambaga M (2016). A new suggestion about existing of membrane - redoxy potential three state line system between donators and acceptors inside the living cells, Asian Journal of Science and technology, Vol.07, Issue, 07, pp.3157-3161.

5. Ambaga M (2016). The buffering capacity of erythrocyte membrane surroundings in relation to free protons, formed in the Full Cycle of Proton and Electron Conductance inside the Human Body. International Journal of Development Research, Vol 06, Issue, 07, pp. 8458-8461.

6. Ambaga M (2016). The Full Cycle of Proton and Electron Conductance inside the Human Body and triple Rlung, Mkhris, Badgan theory of Tibetian Traditional medicine, International Journal of Current Research, Vol 8, Issue 08, p.36391-36393.

7. Ambaga M (2016). The possibility to drive the membrane - redox potential, a three state line system dependent - full 9 stepped cycle of proton conductance inside human body to favorable direction during pathological situations., International Journal of Current Research, Vol, Issue,11, pp 42456-42459, November.

8. Nick Lane, and William F. Martin ( 2012), The origin of membrane bioenergetics J.cell, http://dx.doi.org/10.1016/j.cell.2012.11.050.

9. Víctor Sojo, Andrew Pomiankowski, Nick Lane (2014), A Bioenergetic Basis for Membrane Divergence in Archaea and Bacteria, Published: August 12, 2014, http://dx.doi.org/10.1371/journal.pbio.1001926

10. Filipa L. Sousa, Thorsten Thiergart, Giddy Landan, Shijulal Nelson-Sathi, Inês A. C. Pereira, John F. Allen, Nick Lane, William F. Martin (2013), Early bioenergetic evolution, Published 10 June 2013.DOI: $10.1098 /$ rstb.2013.0088. 